\title{
Meridianbeobachtungen des Planeten (6) Hebe
} am Karlsruher Meridiankreise von $84 \mathrm{~mm}$ Oeffnung.

\begin{tabular}{|c|c|c|c|c|c|c|c|c|c|}
\hline \multicolumn{2}{|c|}{1893} & M. Z. Karlsruhe & \multicolumn{2}{|r|}{$\alpha$ app. } & \multicolumn{2}{|c|}{8 app. } & Parallaxe & \multicolumn{2}{|c|}{$B-R$} \\
\hline Oct. & I 3 & I $2^{\mathrm{h}} 26^{\mathrm{m}} 53^{\mathrm{s}}$ & 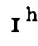 & $57^{\mathrm{m}} 55^{5} \times 3$ & $-16^{\circ}$ & I $39^{\prime \prime} 3$ & $+8 . \circ$ & -0.26 & -8.5 \\
\hline 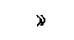 & 19 & II $\quad 5^{8} \quad 54$ & $\mathbf{I}$ & $53 \quad 30.85$ & -- I 7 & 335.7 & +8.0 & -0.19 & -9.6 \\
\hline 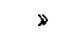 & 20 & $1154 \quad 13$ & $\mathbf{I}$ & $5^{2} 45.98$ & - I 7 & ro 23.9 & +8.0 & -0.20 & -9.1 \\
\hline Nov. & 8 & $10265^{8}$ & $\mathbf{I}$ & $40 \quad 10.74$ & -17 & $51 \quad 15.1$ & $+7 \cdot 5$ & -0.05 & -7.1 \\
\hline * & IO & $10 \quad 189$ & $\mathbf{I}$ & $39 \times 3.5 \mathrm{I}$ & -17 & $45 \quad 5^{I} \cdot 5$ & +7.4 & -0.03 & -6.5 \\
\hline$\gg$ & 12 & 10926 & $\mathbf{I}$ & $3^{8} \quad 22.35$ & -17 & $3^{8} \quad 4^{6.7}$ & +7.3 & -0.10 & $-7 \cdot 5$ \\
\hline
\end{tabular}

Die Grössen B $-R$ bedeuten die Correction der Ephemeride von R. Luther in A. N. 3 r89.

Die Positionen beziehen sich auf den vorläufigen Fundamentalcatalog für die südlichen Zonen der Astronomischen Gesellschaft, dessen Nummern 17, 18, 24, 27 fuir die Ableitung des Aequatorpunktes auf dem Kreise ausschliesslich, für die der Uhrcorrection neben den Anhaltsternen unserer Zone $\left(0^{\circ}\right.$ bis $\left.-8^{\circ}\right)$ verwendet wurden. Oct. 19, Planet $8.0 \%$; Oct. 20, Planet äusserst schwach;

Heidelberg, Königstuhl, I 896 October.
Nov. 10, Planet schwach; Nov. 12, Planet recht schwach. $\mathrm{Da}$ der Meridiankreis bei mittlerer Feldbeleuchtung Sterne 8.0 noch gut zeigt, so war der Planet, wie vorstehende Bemerkungen beweisen, wenn man seinen tiefen Stand in Rücksicht zieht, jedenfalls nicht weit über $8^{\mathrm{m}} \circ$, so dass die Helligkeitsangabe $7 \mathbf{m}_{\mathbf{2}}$ der Ephemeride zu hoch erscheint.

\section{Beobachtungen des Planeten (209) Dido}

angestellt am Repsold'schen Mikrometer des ra inch Aequatoreals von Grubb.

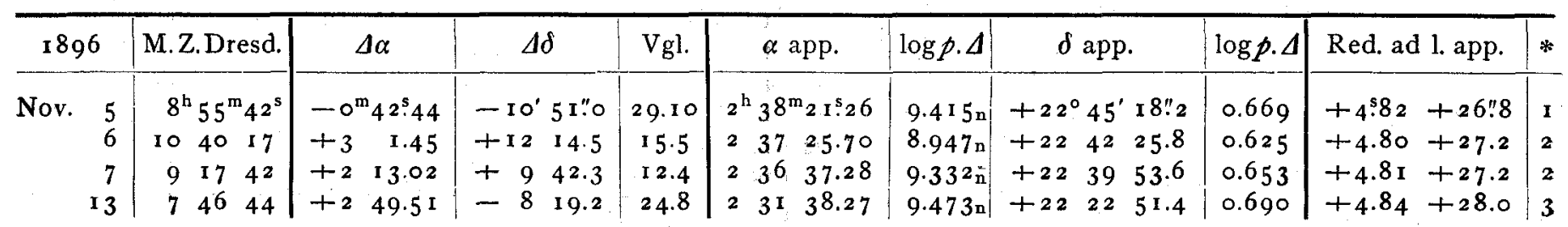

Mittlere Oerter der Vergleichsterne.

\begin{tabular}{|c|c|c|c|}
\hline$*$ & $\alpha I 896.0$ & $\delta 1896.0$ & Autorität \\
\hline I & $2^{\mathrm{h}} 3^{8^{\mathrm{m}}} 5^{8.888}$ & $+22^{\circ} 55^{\prime} 42^{\prime \prime} 4$ & AG. Berlin B. $82 \mathrm{I}$ \\
\hline 2 & $2 \quad 34 \quad 19.44$ & +222944.1 & AG. Berlin B. $80 \mathrm{r}$ \\
\hline 3 & $\begin{array}{lll}2 & 28 & 43.92\end{array}$ & +223042.6 & AG. Berlin B. 781 \\
\hline
\end{tabular}

Die Bilder waren nicht gut. Der Planet ist I 2.5 Grösse.

Dresden, 1896 Nov. 28 .

\section{F. Ristenpart.}

Correction der Ephemeride A. N. $33^{8} 3$.

\begin{tabular}{|c|c|c|c|}
\hline \multicolumn{2}{|c|}{1896} & $\Delta \alpha$ & $\Delta 8$ \\
\hline Nov. & 5 & +1.48 & + + 11.6 \\
\hline & 6 & +1.36 & +8.3 \\
\hline & 7 & $+1 \cdot 3^{8}$ & +8.7 \\
\hline & 13 & +1.35 & +10.8 \\
\hline
\end{tabular}

\section{Beobachtungen von Cometen}

angestellt am. Heliometer der Sternwarte zu Königsberg von Dr. Fritz Cohn.

\begin{tabular}{l|l|l|l|l|l|l|l|l|l|l}
\hline 1896 & M. Z. Kön. & $\Delta \alpha$ & $\Delta \delta$ & Vgl. & $\alpha$ app. & $\log p . \Delta$ & $\delta$ app. & $\log p . \Delta$ & Red. ad I.app. & $*$ \\
\hline
\end{tabular}

Comet 1895 IV.

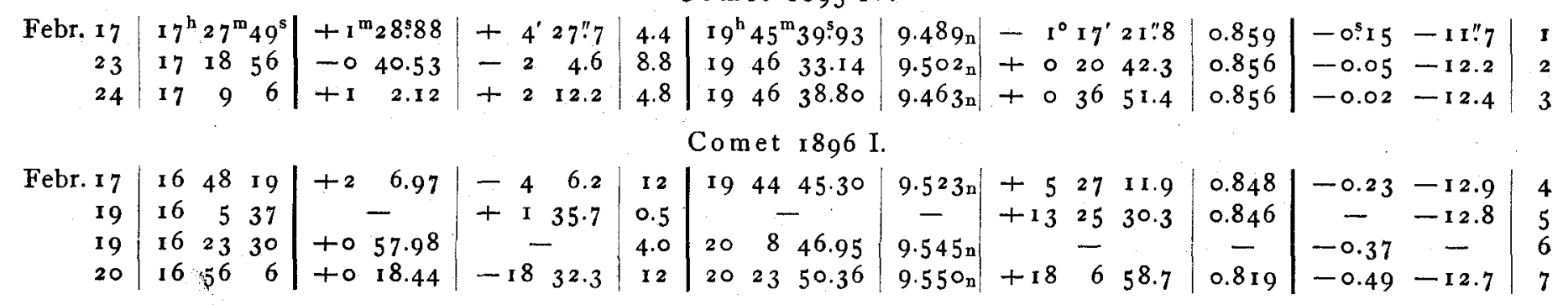

